\title{
Identification of Histomonas meleagridis by in vitro microculture and polymerase chain reaction
}

This article was published in the following Dove Press journal:

Reports in Parasitology

9 June 2011

Number of times this article has been viewed

\author{
Wei Liu',* \\ Jun-yu Peng ${ }^{2, *}$ \\ Fen $\mathrm{Li}^{1}$ \\ Hong-yan Sun ${ }^{3}$ \\ Ying Ding' \\ jing $\mathrm{He}^{\prime}$ \\ Yi Liu'
}

'College of Veterinary Medicine, Hunan Agricultural University,

Changsha; ${ }^{2} \mathrm{NeX}$ Eco-Agriculture Technologies, Inc, Chenzhou, Hunan; ${ }^{3}$ Key Laboratory of Applied Marine Biology of Guangdong Province and Chinese Academy of Sciences, South China Sea Institute of Oceanology, Chinese Academy of Sciences, Guangzhou, People's Republic of China

*These authors contributed equally to this work
Background: In this study, methods for the in vitro microculture of Histomonas meleagridis were defined to lay the foundation for further study of its diagnosis, life cycle, and pathogenic mechanism.

Methods: After serial dilutions of $H$. meleagridis, a single parasite was taken and cultured at $40^{\circ} \mathrm{C}$. Its proliferation was checked by microscopy, and an $18 \mathrm{~S}$ rRNA gene fragment of $H$. meleagridis was amplified using specific primers $(\mathrm{Hmf} / \mathrm{Hmr})$ and sequenced. The sequences were assembled manually and aligned using the Clustal X 1.81 program.

Results: Two tubes showed proliferation of parasites and, after infection with parasites from these tubes, acute histomoniasis was rapidly induced in turkeys. 18S rRNA gene fragments of the two strains were 492 and 495 base pairs in length, respectively, and the sequence homologies with known $H$. meleagridis sequences were both $99.4 \%$. Sequence homology between these two strains was $99.6 \%$, and the variation between the two sequences and Trichomonas was more than $20 \%$.

Conclusion: We conclude that microculture can produce high-purity and highly pathogenic H. meleagridis.

Keywords: Histomonas meleagridis, microculture technology, polymerase chain reaction, identification

\section{Introduction}

Histomoniasis is an acute protozoal disease causing cecal and liver damage, as well as functional disorders, in Galliformes birds. ${ }^{1}$ Histomonas meleagridis is a single-flagellum, parasitic protozoa causing cecal hepatitis. The major invasion sites are the cecum and the liver, and a blood circulation disorder appears in the later stages of the disease, causing the sick bird to develop a purple head, ie, blackhead disease. In recent decades, histomoniasis has caused serious harm to the poultry industry, particularly among turkeys. ${ }^{2}$ It is reported that blackhead disease almost completely destroyed the turkey industry in the Eastern and Midwestern areas of the US in the 1930s. In 1945, blackhead disease accounted for $32.2 \%$ of the total mortality of turkeys in North Carolina. ${ }^{3}$ Susceptibility of other fowl is not high, but the disease causes growth retardation, egg drop, and hinders the healthy development of birds, resulting in enormous economic loss. Through artificial modeling of physiologic conditions to allow the parasite to complete its growth and development phase in vitro, the problem of lack of parasite material for $H$. meleagridis research can now be solved. So far, many researchers have successfully cultured $H$. meleagridis in vitro ${ }^{4-6}$ but using methods that were unable to obtain a pure culture of the parasite, due to contamination with bacteria.
Correspondence: Yi Liu

College of Veterinary Medicine, Hunan

Agricultural University, Changsha 4I0I28,

People's Republic of China

$\mathrm{Tel}+73 \mid 84618090$

$\mathrm{Fax}+73184673618$

Email yiliupro@I63.com 
H. meleagridis causes clinical symptoms similar to those of Trichomonas. It is difficult to distinguish between the two parasites merely on the basis of morphology and host origin. With the development of molecular methods, more satisfactory results have been obtained by genetic markers. The 18S rRNA gene is highly conserved, and easy-to-use universal primers have been widely used in fauna taxonomy studies). ${ }^{7-9}$ Many studies have shown that $18 \mathrm{~S}$ rDNA is an effective molecular marker for studies of the evolutionary mechanism of parasites and for system reconstruction, and the gene is usually one of the molecular markers chosen. ${ }^{10}$ The 18S rRNA gene is a good molecular marker, not only for systematic evolution and biogeography studies of H. meleagridis, but also for its rapid and accurate identification and classification, which is consistent in a number of reports. ${ }^{11-16}$

In the present study, conducted in a Hunan laboratory, we optimized in vitro microculture sample preparation, purification, and proliferation methods to obtain a pure culture of H. meleagridis. Polymerase chain reaction (PCR) amplification of the $18 \mathrm{~S}$ rRNA sequence was then analyzed. This study not only adds to the research data on in vitro culture of H. meleagridis, but also lays the foundation for more in-depth studies for future classification, differential diagnosis, and molecular epidemiology, as well as prevention and treatment of this parasitic disease.

\section{Materials and methods Sampling}

H. meleagridis was isolated from the ceca and livers of infected turkeys in Hunan after a field outbreak. At postmortem, the organs showed gross lesions typical for histomoniasis and were examined to determine the specificity of the methods used (Figure 1). Healthy live turkeys were then artificially infected with embryonated Heterarkis eggs. After presentation of histomoniasis, their cecal contents were cultured in vitro to obtain $H$. meleagridis parasites. Tissue scraped from the cecum and cecal wall of the turkeys that had died of histomoniasis were screen-filtered, placed in a centrifuge tube (Takara Holdings, Kyoto, Japan), diluted by 10 -fold using M-199 culture solution and preheated to $37^{\circ} \mathrm{C}$. After centrifugation for 5 minutes at $2000 \mathrm{rpm}$, the supernatant was pipetted and discarded, then five times the volume of the culture solution was added and homogenized thoroughly. The tube was rested at $37^{\circ} \mathrm{C}$ for $5-10$ minutes, then centrifuged for a further 5 minutes at $2000 \mathrm{rpm}$, after which the supernatant was pipetted and abandoned. This

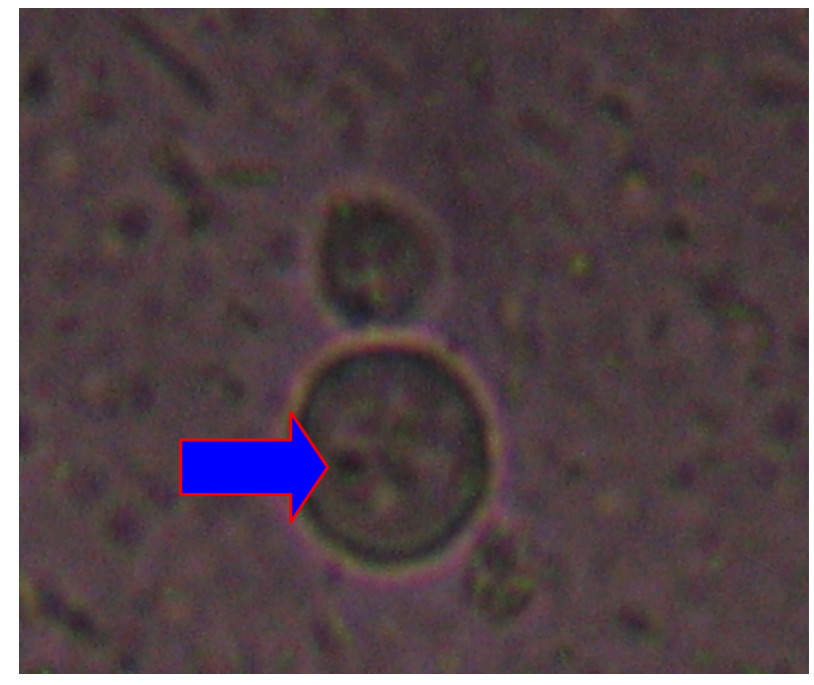

Figure I Histomonas meleagridis in M-199 medium.

procedure was repeated three times in succession, after which the sediment in the tube was transferred to a culture flask. ${ }^{17}$

\section{Preparation of M-I 99 culture medium}

In vitro culture of $H$. meleagridis was done in accordance with the manufacturing instructions for M-199 medium powder (Gibco Co, Grand Island, NY) and stored at $4{ }^{\circ} \mathrm{C}$, whereby 9 mL of M-199 culture medium was pipetted into a sterile cell culture bottle, and $11 \mathrm{mg}$ of sterile rice flour and 15\% fetal calf serum was added. The medium was supplemented with penicillin $200 \mathrm{IU} \mathrm{mL} \mathrm{m}^{-1}$ (Pfizer, New York, NY), streptomycin $200 \mu \mathrm{g} / \mathrm{mL}$ (Pfizer), and amphotericin B $2.5 \mu \mathrm{g} / \mathrm{mL}$ (Promega, Fitchburg, WI).

\section{In vitro microculture of $H$. meleagridis}

Liver and cecal samples from dead $H$. meleagridis-infected turkeys were placed in a $40^{\circ} \mathrm{C}$ temperature-controlled water bath, and the cecum was cut open with sterile scissors. All cecal contents were added to cell culture bottles containing the culture medium and incubated at $40^{\circ} \mathrm{C}$. Using a sterile straw, a drop of culture medium was placed onto a glass slide, immediately covered with a cover slip, and placed under a microscope for examination at 10-40× magnification. If activity of $H$. meleagridis was observable, we made a glass micropipette by clamping an acid silica capillary suction tube with pipette pliers so that the outside diameter was reduced to $25 \mathrm{~mm}$. The micropipette remained fixed on the microscope and the glass pipette was connected by suction via a hose to an empty syringe at one end. The culture medium containing H. meleagridis was 
diluted 1:100 with fresh culture medium so that individual parasites could be seen. A few drops of the $100 \mu \mathrm{L}$ culture medium was placed onto a slide using a straw and added to the microcentrifuge tubes. The process was monitored under a microscope at $400 \times$ magnification in order to ensure that only one parasite was transferred each time. One milliliter of fresh M-199 culture medium was added to each microcentrifuge tube and incubated at $40^{\circ} \mathrm{C}$ for 4 days. Only short periods of cultivation are possible using this process, so $1 \mathrm{~mL}$ of culture solution containing $H$. meleagridis in $9 \mathrm{~mL}$ of fresh M-199 culture solution was required every 2-3 days. Therefore, the pathogenicity of $H$. meleagridis cultivated using this method was lost. The worm bodies of $H$. meleagridis propagate by binary division into different sizes, are amoebiform, and with flagellum, as observed under electron microscopy observation. $H$. meleagridis can be observed in the stained cecum cells. ${ }^{18}$

At 2-4 days, the growth of the parasite was observed with an optical microscope. Ten successive centrifuge tubes were prepared and each tube was observed daily. The growth of $H$. meleagridis was recorded, and cultures were subcultured once every 2 days. Specific primers were used for PCR amplification to detect $H$. meleagridis in each centrifuge tube. All positive tubes were subcultured.

\section{DNA isolation and PCR amplification}

Two hundred microliters of M-199 solution containing $H$. meleagridis was transferred into a $1.5 \mathrm{~mL}$ Eppendorf tube. After centrifugation at $5000 \mathrm{rpm}$ for 5 minutes, the upper $100 \mu \mathrm{L}$ of supernatant was discarded. The sample was added to a tube containing $200 \mu \mathrm{L}$ of nuclear lysis solution (50 $\mu \mathrm{L} 0.5 \mu \mathrm{L}$ ethylenediamine tetra-acetic acid [pH 8.0], and $20 \mu \mathrm{L}$ proteinase $\mathrm{K}[50 \mu \mathrm{g} / \mu \mathrm{L}]$ ) (Merck, Whitehouse Station, NJ). After mixing, the sample was incubated at $55^{\circ} \mathrm{C}$ for 16-18 hours in a temperature-controlled bath to lyse the parasites. Genome DNA was extracted from parasites by SDS/proteinase K treatment, column-purified (Wizard ${ }^{\circledR} \mathrm{SV}$ Genomic DNA Purification System; Promega, Madison, WI) and eluted into $60 \mu \mathrm{L} \mathrm{H_{2 }} \mathrm{O}$ according to the manufacturer's recommendations. ${ }^{19,20}$

Specific primers (Hmf and Hmr) were synthesized using the genome sequence (Genbank accession number AF293056). ${ }^{21}$ Nucleotide sequences were as follows: Hmf 5'-GAAAGCATCTATCAAGTGGAA-3' (forward) and Hmr 5'-GATCTTTTCAAAT TAGCTTTAAA-3' (reverse).

The PCR mixture $(25 \mu \mathrm{L})$ contained $4 \mathrm{mM}$ of $\mathrm{MgCl}_{2}$, $5 \mu \mathrm{M}$ of each primer, $2.5 \mu \mathrm{L} 10 \times$ rTaq buffer, $0.2 \mathrm{mM}$ of each
dNTPs, 1.25 U of rTaq DNA polymerase (Takara Holdings), and $2 \mu \mathrm{L}$ of DNA sample. PCR was performed in a thermocycler (Biometra, Goettingen, Germany) whereby the sample was maintained at $95^{\circ} \mathrm{C}$ for 15 minutes followed by 38 cycles at $94^{\circ} \mathrm{C}$ for 30 seconds (denaturation), at $55^{\circ} \mathrm{C}$ for 1 minute (annealing), and at $72^{\circ} \mathrm{C}$ for 1 minute (extension), followed by a final extension at $72^{\circ} \mathrm{C}$ for 10 minutes.

\section{Purification, cloning, and sequencing of the H. meleagridis $\mathrm{PCR}$ product}

The PCR product was purified using a DNA gel extraction kit, and cloned into E. coli (JM109) using the pGEM $^{\circledR}$-T Easy Vector system (Promega). Transformed cells were selected by overnight incubation at $37^{\circ} \mathrm{C}$ on LB/amp/x-gal/ IPTG plates. Positive recombinant clones were picked and incubated in $1 \mathrm{~mL}$ of Terrific Broth (in the presence of $50 \mu \mathrm{g} / \mathrm{mL}$ ampicillin) overnight at $37^{\circ} \mathrm{C}$ with shaking. Positive recombinant clones were sequenced.

\section{Sequencing}

Positive clones were sent to Nanjing Jinsite Biotechnology Co for sequencing. Sequences were then assembled manually and aligned using the program Clustal X 1.81 (Clustal, Dublin, Ireland) 22 and DNAstar 5.0 software (DNASTAR, Inc, Madison, WI). Pairwise comparisons were made based on the level of sequence differences $(D)$ using the formula $D=1-(M / L)$, where $M$ is the number of alignment positions at which the two sequences have a base in common, and $L$ is the total number of alignment positions over which the two sequences are compared. ${ }^{23,24}$ The $18 \mathrm{~S}$ rRNA gene sequences were aligned with references in GenBank (Accession No AJ920323) using Clustal X 1.81, ${ }^{22}$ with manual adjustment.

\section{Results}

\section{In vitro culture}

Liver or cecal contents from dead turkeys suspected of harboring histomoniasis infection were added to fresh M-199 medium and cultured at $40^{\circ} \mathrm{C}$ in incubators. After 48 hours, microscopic observation at 10-40× amplification revealed many vacuolar, Histomonas meleagridis-like amorphous organisms, of size 5-30 $\mu \mathrm{m}$, continuously carrying out pendulum-like movements (Figure 2).

\section{In vitro microculture}

Culture medium containing $H$. meleagridis was gradually diluted until only one parasite could be seen in the field of 
A

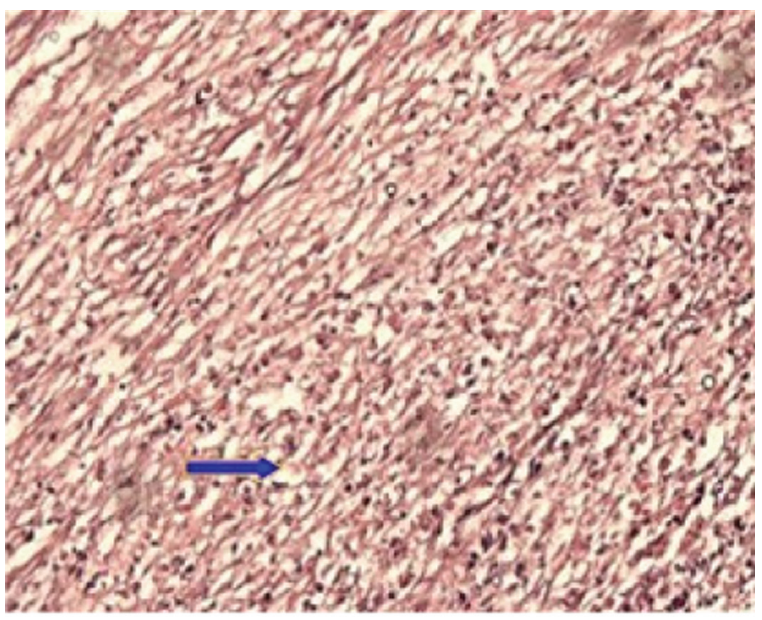

B

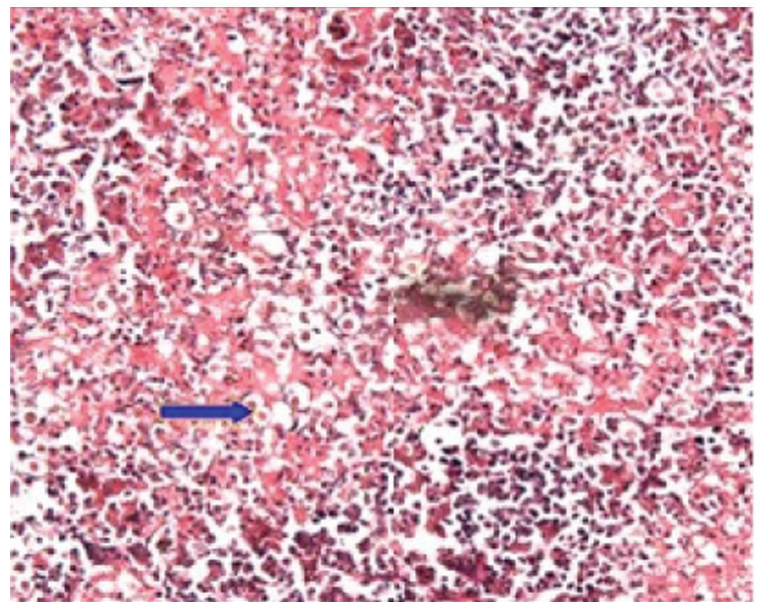

Figure 2 Histological section of the cecum and liver from infected turkeys (A, B)

vision under the microscope. Using a capillary suction tube, this parasite was transferred into $1 \mathrm{~mL}$ of M-199 culture medium in a $1.5 \mathrm{~mL}$ centrifuge tube, cultured at $40^{\circ} \mathrm{C}$ in an incubator, and observed under the microscope daily. The growth of $H$. meleagridis in the centrifuge tube was recorded and subcultured once every 2 days. After 48 hours, proliferation of $H$. meleagridis was observed in only two of the 10 sample microculture tubes.

\section{PCR identification of microcultured}

\section{H. meleagridis}

Two hundred microliters of culture medium were taken from each of the 10 microculture tubes and digested. Samples 1

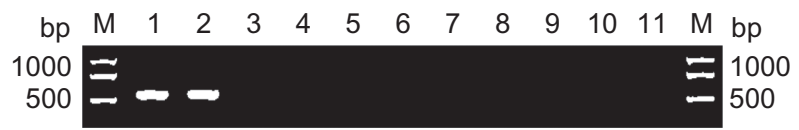

Figure 3 Electrophoresis of polymerase chain reaction products from microculture of Histomonas meleagridis. M. DL-2000 DNA marker; I-I0. $\mathrm{HM}_{1}-\mathrm{HM}_{10}$; II, negative control.
Table I Results of microculture of Histomonas meleagridis

\begin{tabular}{lcccccccccc}
\hline Number & I & $\mathbf{2}$ & $\mathbf{3}$ & $\mathbf{4}$ & $\mathbf{5}$ & $\mathbf{6}$ & $\mathbf{7}$ & $\mathbf{8}$ & $\mathbf{9}$ & $\mathbf{1 0}$ \\
\hline 24 hours & - & - & - & - & - & - & - & + & + & - \\
48 hours & - & - & - & - & - & - & - & + & + & - \\
\hline
\end{tabular}

and 2 in Figure 3 correspond to samples 8 and 9 in Table 1. DNA was extracted and amplified using specific primers. The PCR results are shown in Figure 3.

\section{PCR identification of the $18 \mathrm{~S}$ rRNA gene sequence}

The two successfully amplified fragments were cloned and the positive transformants were selected for PCR identification. The HM1 and HM2 strain DNA fragments were about 550 base pairs. No bands were observed for the negative control (see Figure 4).

\section{Sequencing results and analysis}

Sequencing results showed that the $18 \mathrm{~S}$ rRNA gene sequences of the two samples were 536 and 539 base pairs (GenBank Accession numbers GQ225771 and GQ225772, respectively). Comparisons between the sample sequences and the corresponding sequence of H. meleagridis in GenBank (Accession number AJ920323) using DNAstar 5.0 showed similarity between the two samples was over $97.6 \%$ and the mutated rate was $1.4 \%-2.4 \%$. Similarity between the two H. meleagridis strains was $98.6 \%$ and comparison with Tetratrichomonas gallinarum and Tritrichomonas foetus in GenBank showed that the similarity with these organisms was less than $81.2 \%$. Neighbor joining and maximum parsimony were used for phylogenetic analysis, and carried out using the PAUP 4.0 Beta10 program (Sinauer Associates, Inc, Sunderland, MA). ${ }^{25}$ The consensus tree was obtained after bootstrap analysis. With 1000 replications, values above $50 \%$ were obtained (Figure 5), and the two samples were highly bootstrapped.

\section{Discussion}

In this study, $10 \mathrm{H}$. meleagridis strains were isolated, but normal parasite proliferation was found only in two samples,

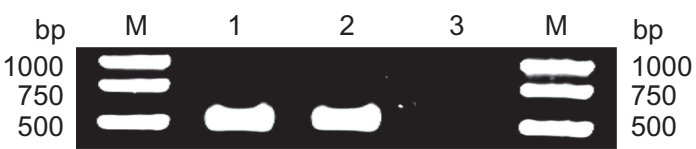

Figure 4 Analysis of polymerase chain reaction-amplified I8S rRNA gene from Histomonas meleagridis by agarose gel electrophoresis M, DL2000 DNA marker; I HMI, 2 HM2, and 3 negative control. 


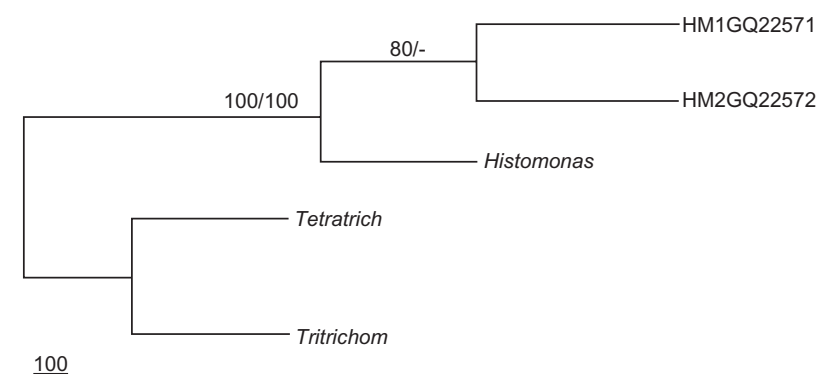

Figure 5 The Bootstrap consensus phylogram of the stationary tree reconstructed by maximum parsimony using the I8S rRNA gene sequences.*

Note: *Numbers at nodes indicate bootstrap values resulting from neighbor joining/ maximum parsimony.

representing a success rate of only $20 \%$, partly because of the weak resistance of $H$. meleagridis to the external environment, and also because of the lack of a capsule to protect the parasite. In addition, death of the parasite may have occurred during the separation process or as a result of adherence of organisms to the wall of the straw, preventing transfer to the centrifuge tube. It is therefore difficult to culture a single parasite. Here, we created a suitable environment for survival when carrying out micromanipulation of the parasites to separate them. Two groups of turkeys were inoculated with the pure $H$. meleagridis via the cloaca. Fourteen to 18 days after infection, all turkeys suffered from acute histomoniasis and died. It was found that the pathologic changes in their livers and ceca were exactly the same as those seen with histomoniasis, indicating $H$. meleagridis successfully proliferated in two tube samples, and their original pathogenicity was retained. Specific PCR products were obtained from liver samples in both groups, while only an amplification product was observed in the first cecal sample. Specific PCR amplification products were obtained from both the cecum and liver in work by Hess and Lund, and other research groups who have infected turkeys with $H$. meleagridis cultured in vitro. ${ }^{26,27}$ Low levels of $H$. meleagridis in the cecum were observed because of weak virulence of $H$. meleagridis microcultured in the second tube and their reduced invasion of the cecum.

Since the discovery of $H$. meleagridis in 1895 , researchers have used a variety of approaches for the prevention and diagnosis of histomoniasis. Diagnosis is initially made at onset of clinical symptoms and pathologic changes. However, the use of traditional methods of pathogen detection or epidemiologic investigations is time-consuming and not conducive to rapid clinical decision-making. Furthermore, microscopic examination is not suitable for large-scale use. With the extensive use of PCR technology, great progress has been made in the molecular taxonomy of parasites and molecular genetics research. Point mutations in the $18 \mathrm{~S}$ rDNA gene are few and restricted to a certain area, which makes homologous comparison between 18S rDNA genes easier and more informative. The use of molecular methods in this study confirmed $H$. meleagridis infection in commercial turkey stock in Hunan at the molecular level. The results of PCR amplification from microculture are consistent with microscopic observations. Hess et al and Grabensteiner et al have successfully amplified genes directly from culture medium of $H$. meleagridis by the design of different primers, and distinguished it from chicken Trichomonas and yeast. ${ }^{14,28}$ Their results showed that the two $H$. meleagridis strains obtained shared a high similarity (99.4\%) with the corresponding $H$. meleagridis in Genbank. In addition, comparison between the two $H$. meleagridis cultures also showed high similarity (99.6\%). However, the sample sizes are too small, and the geographical distribution is too narrow, so these results do not fully reflect their geographical differences. Through sequence analysis, it was shown that they shared a high sequence similarity with the corresponding sequence of the reported $H$. meleagridis strains, but the similarity with Trichomonas was low, suggesting that the $18 \mathrm{~S}$ rDNA gene is an effective molecular marker for study of the evolution and taxonomy of $H$. meleagridis.

\section{Acknowledgment}

This work was supported by grants from the National Natural Science Foundation of China (3077616).

\section{Disclosure}

The authors report no conflicts of interest in this work.

\section{References}

1. Tyzzer EE, Fabyan F. Further studies on "blackhead" in turkeys, with special reference to transmission by inoculation. Diseases. 1920;27: 207-239.

2. Hinshaw WR. Diseases of Turkeys Bulletin. Berkeley, CA: University of California Agricultural Experiment Station; 1937.

3. Dearstyne RS. When and why do they die? Turkey World. 1945;14: 62-63.

4. Drbohlav AJ. The cultivation of the protozoan of blackhead. J Med Res. 1924;44:677-678

5. Dwyer DM. An improved method for cultivating Histomonas meleagridis. J Parasitol. 1970;56:192-193.

6. Lesser E. In vitro cultivation of Histomonas meleagridis free of demonstrable bacteria. Parasitol. 1961;8:228-230

7. Rohde K, Hefford C, Ellis JT. Contributions to the phylogeny of Platyhelminthes based on partial sequencing of 18S ribosomal DNA. Int J Parasitol. 1993;23:705-724.

8. Bridge D, Cunningham CW, DeSalle R, Buss LW. Class-level relationships in the phylum Cnidaria: Molecular and morphological evidence. Mol Biol Evol. 1995;12:679-689.

9. Medina M, Collins AG, Silberman JD, Sogin ML. Evaluating hypotheses of basal animal phylogeny using complete sequences of large and small subunit rRNA. Proc Natl Acad Sci U S A. 2001;98:9707-9712. 
10. Adam RD. Biology of Giardia lamblia. Clin Microbiol Rev. 2001;14: 447-475.

11. Huber K, Chauve C, Zenner L. Detection of Histomonas meleagridis in turkeys cecal droppings by PCR amplification of the small subunit ribosomal DNA sequence. Vet Parasitol. 2005;131:311-316.

12. Grabensteiner E, Liebhart D, Weissenbock H, Hess M. Broad dissemination of Histomonas meleagridis determined by the detection of nucleic acid in different organs after experimental infection of turkeys and specified pathogen-free chickens using amono-eukaryotic culture of the parasite. Parasitol Int. 2006;55:317-322.

13. Hafez HM, Hauck R, Lüschow D, McDougald L. Comparison of the specificity and sensitivity of PCR, nested PCR, and real-time PCR for the diagnosis of histomoniasis. Avian Dis. 2005;49:366-370.

14. Grabensteiner E, Hess M. PCR for the identification and differentiation of Histomonas meleagridis, Tetratrichomonas gallinarum and Blastocystis spp. Vet Parasitol. 2006;142:223-230.

15. Liebhart D, Weissenböck H, Hess M. In-situ hybridization for the detection and identification of Histomonas meleagridis in tissues. J Comp Pathol. 2006;135:237-242.

16. Mazet M, Diogon M, Alderete JF, Vivarès CP, Delbac F. First molecular characterisation of hydrogenosomes in the protozoan parasite Histomonas meleagridis. Int J Parasitol. 2008;38:177-190.

17. Trager W, Jensen JB. Human malaria parasites in continuous culture. Science. 1976;193:673-675.

18. Harold van der Heijden HM, McDougald LR, Landman WJ. High yield of parasites and prolonged in vitro culture of Histomonas meleagridis. Avian Pathol. 2005;34:505-508.

19. Zhao GH, Mo XH, Zou FC, et al. Genetic variability among Schistosoma japonicum isolates from different endemic regions in China revealed by sequences of three mitochondrial DNA genes. Vet Parasitol. 2009a; 162:67-74.

20. Zhao GH, Li J, Zou FC, et al. ISSR, an effective molecular approach for studying genetic variability among Schistosoma japonicum isolates from different epidemic provinces in mainland China. Infect Genet Evol. 2009b;9:903-907.
21. Gerbod D, Edgcomb VP, Noël C, Zenner L, Wintjens R, Delgado-Viscogliosi P, et al. Phylogenetic position of the trichomonad parasite of turkeys, Histomonas meleagridis (Smith) Tyzzer, inferred from small subunit rRNA sequence. $J$ Eukaryot Microbiol. 2001;48: 498-504.

22. Thompson JD, Gibson TJ, Plewniak F, Jeanmougin F, Higgins DG. The Clustal $\mathrm{X}$ windows interface: Flexible strategies for multiple sequence alignment aided by quality analysis tools. Nucleic Acids Res. 1997;24: 4876-4882.

23. Li MW, Lin RQ, Song HQ, Sani RA, Wu XY, Zhu XQ. Electrophoretic analysis of sequence variability in three mitochondrial DNA regions for ascaridoid parasites of human and animal health significance. Electrophoresis. 2008;29:2912-2917.

24. Zhao GH, Mo XH, Zou FC, et al. Genetic variability among Schistosoma japonicum isolates from different endemic regions in China revealed by sequences of three mitochondrial DNA genes. Vet Parasitol. 2009;162:67-74.

25. PAUP*: Phylogenetic Analysis Using Parsimony (and Other Methods). Sunderland, MA: Sinauer Associates; 2002.

26. Hess M, Grabensteiner E, Liebhart D. Rapid transmission of the protozoan parasite Histomonas meleagridis in turkeys and specific pathogen free chickens following cloacal infection with a mono-eukaryotic culture. Avian Pathol. 2006;35:280-285.

27. Lund EE, Chute AM, Wilkins GC. The wild turkey as a host for Heterakis gallinarum and Histomonas meleagridis. J Wildl Dis. 1975;11:376-81.

28. Hess M, Kolbe T, Grabensteiner E, Prosl H. Clonal cultures of Histomonas meleagridis, Tetratrichomonas gallinarum and a Blastocystis sp established through micromanipulation. Parasitology. 2006;133: $547-554$.
Reports in Parasitology

\section{Publish your work in this journal}

Reports in Parasitology is international, peer-reviewed, open access journal publishing original research, reports, reviews and commentaries on all areas of parasitology. The manuscript management system is completely online and includes a very quick and fair peer-review

\section{Dovepress}

system. Visit http://www.dovepress.com/testimonials.php to read real quotes from published authors. 